\title{
Observation of Time-Domain Modulation of Free-Electron-Laser Pulses by Multipeaked Electron-Energy Spectrum
}

\author{
V. Petrillo, ${ }^{1}$ M. P. Anania, ${ }^{2}$ M. Artioli, ${ }^{3}$ A. Bacci, ${ }^{1}$ M. Bellaveglia, ${ }^{2}$ E. Chiadroni, ${ }^{2}$ A. Cianchi, ${ }^{4}$ F. Ciocci, ${ }^{3}$ G. Dattoli, ${ }^{3}$ \\ D. Di Giovenale, ${ }^{2}$ G. Di Pirro, ${ }^{2}$ M. Ferrario, ${ }^{2}$ G. Gatti, ${ }^{2}$ L. Giannessi, ${ }^{3}$ A. Mostacci, ${ }^{5}$ P. Musumeci, ${ }^{6}$ A. Petralia, ${ }^{3}$ \\ R. Pompili, ${ }^{4}$ M. Quattromini, ${ }^{3}$ J. V. Rau, ${ }^{7}$ C. Ronsivalle, ${ }^{3}$ A. R. Rossi, ${ }^{1}$ E. Sabia, ${ }^{3}$ C. Vaccarezza,${ }^{2}$ and F. Villa ${ }^{2}$ \\ ${ }^{1}$ INFN-Milano and Università di Milano, Via Celoria, 1620133 Milano, Italy \\ ${ }^{2}$ INFN-LNF, Via Enrico Fermi, 4000044 Frascati, Roma, Italy \\ ${ }^{3}$ ENEA Frascati, Via Enrico Fermi, 4500044 Frascati, Roma, Italy \\ ${ }^{4}$ INFN-Roma Tor Vergata and Università di Roma Tor Vergata, Via della Ricerca Scientifica, 100133 Roma, Italy \\ ${ }^{5}$ Università La Sapienza di Roma, Via Antonio Scarpa 14, Roma, Italy \\ ${ }^{6}$ UCLA, Los Angeles, California 90095, USA \\ ${ }^{7}$ ISM-CNR Via del Fosso del Cavaliere, 10000133 Roma, Italy \\ (Received 26 April 2013; published 13 September 2013)
}

\begin{abstract}
We present the experimental demonstration of a new scheme for the generation of ultrashort pulse trains based on free-electron-laser (FEL) emission from a multipeaked electron energy distribution. Two electron beamlets with energy difference larger than the FEL parameter $\rho$ have been generated by illuminating the cathode with two ps-spaced laser pulses, followed by a rotation of the longitudinal phase space by velocity bunching in the linac. The resulting self-amplified spontaneous emission FEL radiation, measured through frequency-resolved optical gating diagnostics, reveals a double-peaked spectrum and a temporally modulated pulse structure.
\end{abstract}

DOI: 10.1103/PhysRevLett.111.114802

PACS numbers: 41.60.Cr, 42.55.-f, 42.65.Ky

Radiation pulses with attosecond to femtosecond time scales represent a real possibility for a breakthrough in science and technology, permitting unprecedented insights into the ultrafast electron and nuclear dynamics [1-3]. The time-resolved study of electron rearrangements could lead to significant advances in the understanding of intermolecular processes, chemical bond breaking and formation, and the interaction of photoactivated molecules with their environment.

Trains of ultrashort radiation pulses enable stroboscopic electron imaging [4] and the investigation of the response accompanying collective electron motion in nanomaterials [5]. They also find further applications in other technical fields, such as the enhancement of transmission or reflectivity in materials, resonant inelastic x-ray scattering, or the $a b$ initio phasing of nanocrystals [6].

Sequences of spikes have been synthesized by means of the high harmonic generation driven by lasers in gases [7] and regularly used in experiments $[4,8]$, but are severely limited in efficiency approaching the $\mathrm{keV}$ range.

Free-electron lasers (FELs) are capable of producing high brightness pulses in the x-ray spectral region [9-12]. The FEL, in the self-amplified spontaneous emission (SASE) mode of operation [13], generates radiation with limited temporal coherence [14], time duration of the order of the electron bunch length and structured in a chaotic succession of random peaks. The typical time scale of these radiation spikes is set by the FEL Pierce parameter $\rho$ [13]. Several techniques have been explored to increase longitudinal coherence, stability, and/or to shorten the FEL pulse time scale towards the attosecond domain. The amplification of one single SASE spike has been demonstrated by compressing the electron beam close or below the FEL coherence length $[15,16]$, by using a chirped bunch energy combined with a matched undulator taper [17-19], or by spoiling the whole electron beam except a limited fraction [20,21], a technique that has also been implemented to produce double pulse two-color radiation for pump and probe experiments [22]. Short single or multiple pulses have also been produced in seeded or cascaded FELs [23-27], with increased coherence and shot to shot stability. More sophisticated seeding concepts $[28,29]$ lead to the possibility of producing single- or two-color subfemtosecond pulses in the soft x-ray's range [30,31], but with similar limitation in terms of gain bandwidth.

Different techniques [32,33], relying on concepts adapted to FELs from mode-locked cavity lasers, have been proposed for reducing the characteristic time scales of x-ray pulses beyond the FEL bandwidth limitation. They foresee the generation of trains of high peak coherent power flashes with large contrast ratio by applying a series of spatiotemporal shifts between the copropagating radiation and the electron bunch.

This Letter presents the experimental demonstration of a novel scheme for the generation of a regular short pulse sequence based on FEL lasing from a two-level electron energy distribution. The experiment was carried out at the SPARC_LAB facility [34,35] in the IR-optical frequency range. 
A double-peaked electron energy spectrum is obtained by the laser comb technique in the velocity bunching [36,37]. The electron beam, constituted by two short and balanced bunches, is extracted from the linac accelerating section near the maximum compression. At this point, the two beamlets are temporally superimposed in the longitudinal phase space but split in energy, and characterized by a different value of the Lorentz factor $\gamma_{i}(i=1,2)$. If the energy difference $\Delta \gamma=\left|\gamma_{1}-\gamma_{2}\right|$ satisfies the condition

$$
\frac{\Delta \gamma}{\gamma}>2 \rho \text {, }
$$

with $\gamma$ being the average value of the electron Lorentz factor, the FEL amplification process for the two distributions takes place independently at the respective resonance wavelengths $\lambda_{i}=\left[\lambda_{u}\left(1+K_{u}^{2} / 2\right) / 2 \gamma_{i}^{2}\right]\left(K_{u}\right.$ is the deflection parameter and $\lambda_{u}$ the period of the undulator).

Furthermore, if the electron beam width $L_{b}$ is shorter than $2 \pi$ times the cooperation length $L_{c}=\lambda / 4 \pi \sqrt{3} \rho$, then the FEL emission occurs in the single spike regime with the output radiation characterized by nearly transform-limited pulses, each with a coherent phase. Consequently, beating fringes are produced in the time domain and a train of regular pulses can be obtained. The total radiation bandwidth is now set by the electron energy separation and not by the FEL parameter $\rho$, thus enabling the formation of very short temporal structures. The Fourier analysis for a spectrally double-peaked radiation pulse yields a sinusoidally varying intensity profile where the time separation of the fringes $\Delta T$ depends on the difference in energy of the bunches,

$$
\Delta T=\frac{\lambda^{2}}{c\left(\lambda_{2}-\lambda_{1}\right)}=\frac{\lambda_{u}\left(1+K_{u}^{2} / 2\right)}{4 c \gamma \Delta \gamma} .
$$

Figure 1 presents the trend of the fringe separation with the energy of the electron beam, with the perspective of reaching the attosecond domain in the x-ray regime. We also show in this same figure the shape of the spectrum and the pulse profile as obtained from GENESIS 1.3 simulations for a number $N$ of spectral lines respectively equal to 2 and 4. In the case of $N$ different energy distributions, the width of the fringes shrinks as $1 / N$, due to a gratinglike effect. Because of the fact that in the SASE process the amplification starts from the initial shot noise, the time position of the interference pattern fluctuates randomly each shot, but not the spacing of the fringes. The extrapolation of the method to the UV-ray or x-ray domain could imply a number of demanding technical challenges concerning the radiation diagnostics and the beam preparation. The compression of the beamlets would suggest the operation at low charge, where the beam dynamics during compression with velocity bunching or magnetic chicane requires a tight optimization and control of the beam line optics, and of nonlinearities such as coherent synchrotron radiation
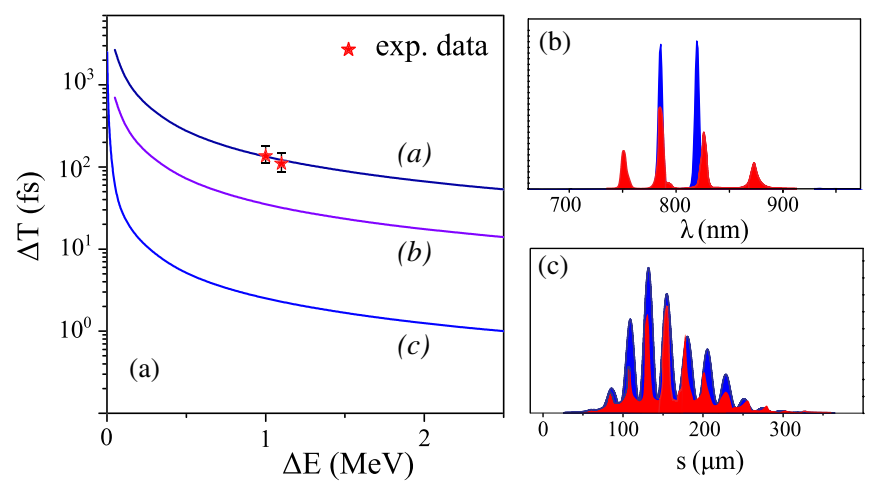

FIG. 1 (color online). (a) Dependence of the fringe separation on the energy distance between the electron packets for $(a)$ SPARC case, $\lambda=800 \mathrm{~nm},(b) \lambda=30 \mathrm{~nm},(c) \lambda=0.15 \mathrm{~nm}$. Spectrum (b) and temporal shape (c) of the pulse, obtained by GENESIS 1.3, for $N=2$ electron packets (blue, dark curve) and $N=4$ (red, light curve) and simulation parameters in the optical range. The width of the fringes scales as $1 / N$.

and wakefields which could lead to misalignment of the bunches or deterioration of their emission properties.

The experiment was performed with the undulator tuned at $\lambda=800 \mathrm{~nm}$ to allow using the spectrotemporal beam diagnostics available at SPARC_LAB. The data were acquired during two experimental shifts in different compression conditions of the electron beam. The experimental parameters are summarized in Table I columns A and B.

The experimental procedure started with the generation of a comb laser beam produced by passing the laser through a birefringent $\alpha$-BBO crystal and characterized and composed by two pulses with time separation of about 4 ps and width of about 100 fs. The resulting, ps-spaced, electron beam was propagated along the linac (three $S$ band cavities) and compressed by means of the velocity bunching method, extensively studied at SPARC [37,38], consisting of the injection of the electrons into the first

TABLE I. Electron beam main parameters (measured values with standard deviation) and radiation characteristics.

\begin{tabular}{lcc}
\hline \hline Quantity & Beam A & Beam B \\
\hline Energy $(\mathrm{MeV})$ & $90.11 \pm 0.06$ & $93.04 \pm 0.10$ \\
Total charge $(\mathrm{pC})$ & $150 \pm 10$ & $165 \pm 10$ \\
Emittance $x(\mathrm{~mm} \mathrm{mrad})$ & $1.56 \pm 0.10$ & $1.68 \pm 0.18$ \\
Emittance $y(\mathrm{~mm} \mathrm{mrad})$ & $1.70 \pm 0.12$ & $1.81 \pm 0.15$ \\
$\begin{array}{l}\text { Energy spread }(\mathrm{MeV}) \\
\text { Energy spread single }\end{array}$ & $0.62 \pm 0.01$ & $0.59 \pm 0.01$ \\
$\quad$ beamlet $(\mathrm{MeV})$ & $0.30 \pm 0.01$ & $0.27 \pm 0.01$ \\
$\begin{array}{l}\text { Energy separation }(\mathrm{MeV}) \\
\text { Time duration }(\mathrm{ps})\end{array}$ & $1.01 \pm 0.11$ & $1.07 \pm 0.14$ \\
$\begin{array}{l}\text { Corrected FEL parameter single } \\
\quad \text { beamlet }\end{array}$ & $0.63 \pm 0.03$ & $0.30 \pm 0.01$ \\
3D cooperation length single & $5 \times 10^{-4}$ & $1.5 \times 10^{-3}$ \\
$\quad$ beamlet $(\mu \mathrm{m})$ & 36 & 12.5 \\
\hline \hline
\end{tabular}




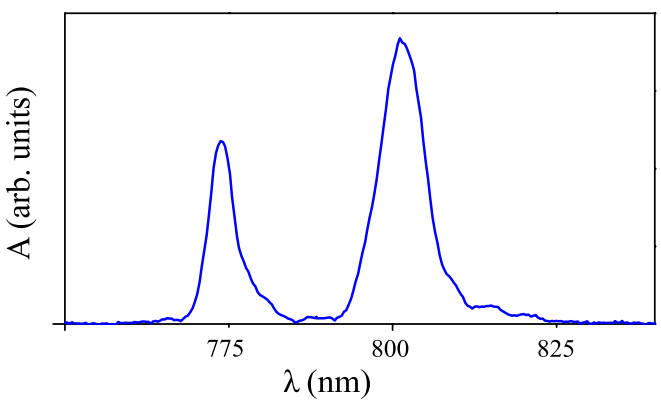

FIG. 2 (color online). Single-shot two-color spectra taken with the fiber spectrometer (resolution: $1.2 \mathrm{~nm}$ at $800 \mathrm{~nm}$ ).

accelerating structure close to the zero crossing rf field phase. Since, at this stage, the beam velocity is much lower than the phase velocity of the rf wave, the electrons slip back to phases where the field is accelerating, being chirped and compressed at the same time. The method has been applied not only with one single electron bunch, but also for the controlled compression of sequences of pulses [39].

Subsequently, the electron beam was injected into the undulator system, consisting of six sections of 75 periods, with $\lambda_{u}=2.8 \mathrm{~cm} \mathrm{[40].} \mathrm{The} \mathrm{optimum} \mathrm{matching} \mathrm{condition}$ of the electron beam to the undulator was found by using the average values of energy and projected Twiss parameters.

The FEL radiation was characterized by means of a joulemeter, a fiber spectrometer, and a near-infrared (NIR) grating-eliminated no-nonsense observation of ultrafast incident laser light e-fields (GRENOUILLE) frequency-resolved optical gating (FROG) for the direct detection of the time-domain pulse structure. The transverse profile of the radiation pulse was regular and symmetric with a single maximum on axis. The observation of a double spectral peak structure on the spectrometer (see Fig. 2) and on FROG in a large fraction of the shots was the demonstration that both electron beams were lasing contemporaneously. The signal level, as measured by the Joulemeter, was always above $30 \mu \mathrm{J}$, indicating that we were close to saturation.

In the first experimental condition (Table I, column A), the linac rf phases were set to extract the electron beam before the condition of maximum compression, when the rotation in the phase space, responsible for the energy and temporal separation of the two beams, was not completed. The experimental longitudinal phase space of the electrons, shown in Fig. 3(a), was measured using a transverse deflecting cavity and a dispersing dipole. The partial current profiles of both bunches are shown together with the total one in Fig. 3(b). The beamlets are overlapping only in the central part. The whole beam has a peak current of about $110 \mathrm{~A}$, and $630 \pm 30 \mathrm{fs} r m s$ pulse duration at energies of $90 \mathrm{MeV}$. The $1 \mathrm{D}$ FEL parameter for each single beamlet is $\rho \approx 4.1 \times 10^{-3}$. Taking into account the
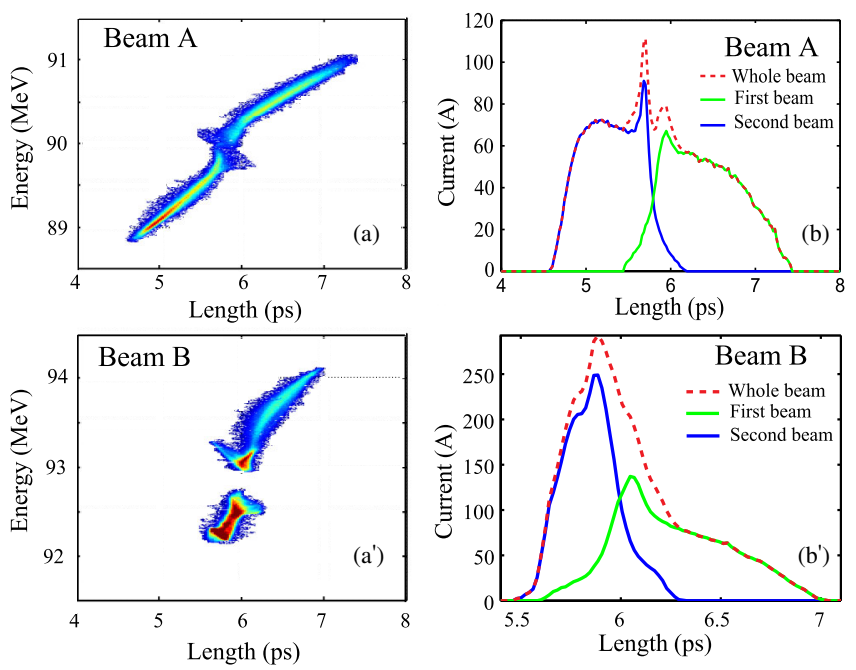

FIG. 3 (color online). Top row: (a) experimental electron phase space and (b) current profiles in the case of beam A. Bottom row: same as top row but for beam B.

$0.3 \mathrm{MeV}$ beamlet energy spread and 3D effects, the corrected FEL parameter is $\rho=5 \times 10^{-4}$, with a corresponding cooperation length $L_{c}=36 \mu \mathrm{m}$. These parameters satisfy the single spike condition $L_{b} \lesssim 2 \pi L_{c}$.

An example of spectral measurements is shown in the first row of Fig. 4(a), where the spectrometer (blue, dark curve) and the FROG (red, light curve) signals are
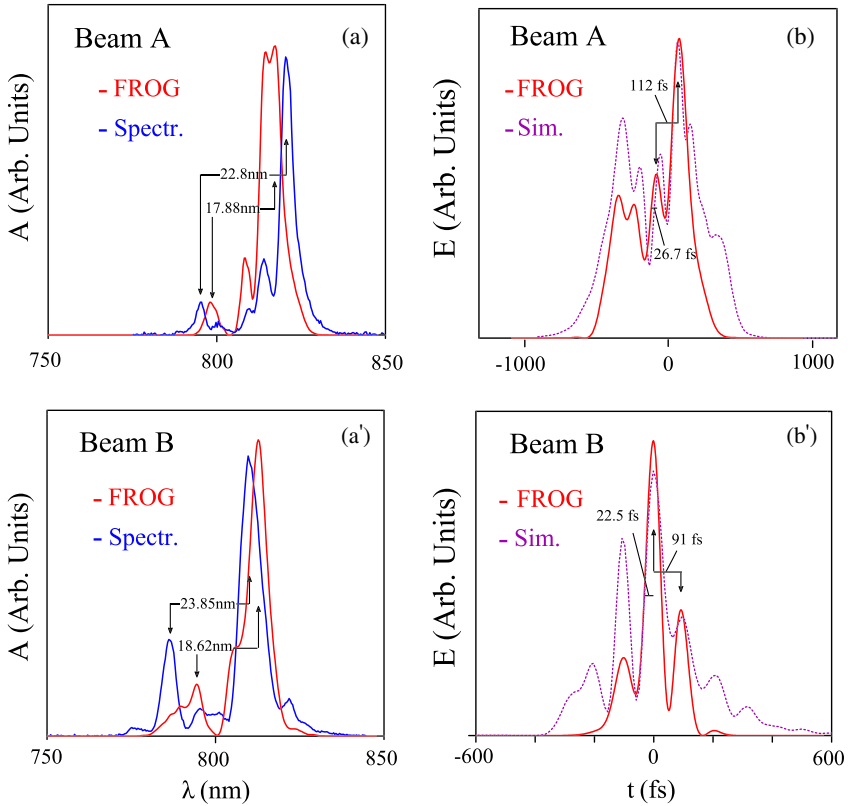

FIG. 4 (color online). Top row, beam A: (a) FROG spectral signal (red, light curve) and spectrometer signal (blue curve, presenting an offset of $10 \mathrm{~nm}$ with respect to the FROG trace). (b) Temporal pulse $E$ from FROG (blue, dark line) and start to end simulation by T-STEP and GENESIS 1.3 (violet dotted curve). Bottom row: same as for beam A but for beam B. Wavelength interdistances are evaluated between the arrows. 

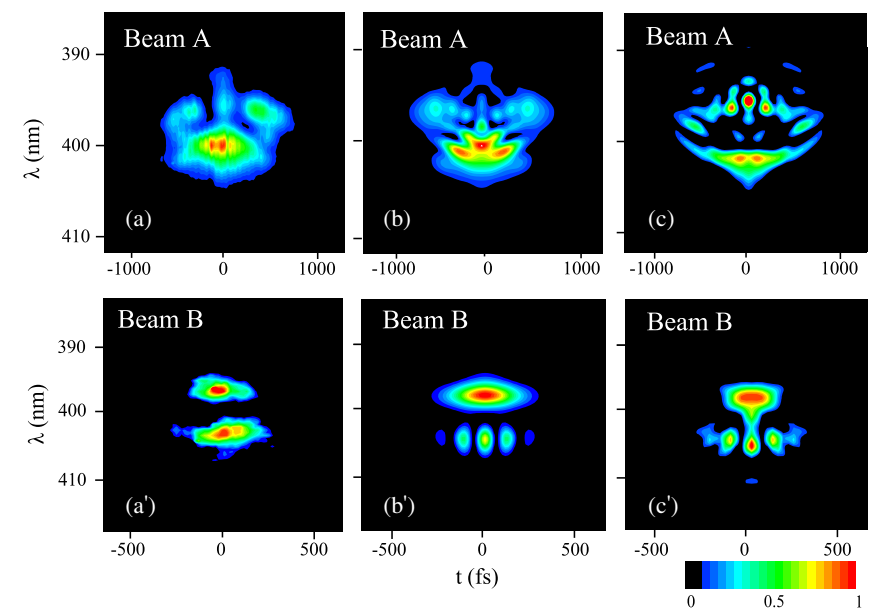

FIG. 5 (color online). Top row: (a) experimental, (b) retrieved, and (c) simulated FROG traces in the case of beam A. Bottom row: same as for beam A but for beam B. FROG near-infrared GRENOUILLE: time-bandwidth product $<10$; spectral resolution $0.7 \mathrm{~nm}$ at $800 \mathrm{~nm}$; single-shot sensitivity $1 \mu \mathrm{J}$.

presented. The wavelength interdistance observed with the spectrometer was $\Delta \lambda=22.8 \mathrm{~nm}$, corresponding to an energy difference $\Delta E=1.3 \mathrm{MeV}$. In $90 \%$ of the shots, the spectrum was double peaked with a preponderance of the power in the larger wavelength band, that sometimes presents structures probably due to SASE fluctuations. The analysis of the FROG traces reveals two neat spectral lines with a distance $\Delta \lambda=17.88 \mathrm{~nm}$, corresponding to an energy gap of $\Delta E=1.03 \mathrm{MeV}$. The first row of Fig. 5 shows one of the experimental FROG traces [5(a)] and the retrieved result [Fig. 5(b)], that present a satisfactory overall agreement. The measure was compared with the output of start-to-end simulations performed using T-STEP [41] and GENESIS 1.3 [42] [Fig. 5(c)], finding similar key features. The temporal structure of the pulse as given by the FROG setup [first row of Fig. 4, red solid curve of 4(b)] is in reasonable agreement with the GENESIS 1.3 simulation (dotted black curve). Interference fringes are visible in the central part of the pulse where a residual superposition of the two bunches is present. The interdistance was $\Delta T=$ $112 \mathrm{fs}$, in agreement with the measured energy separation as shown in Fig. 1. The rms width of each of the fringes $\Delta t_{f, \mathrm{rms}}=26.7 \mathrm{fs}$ is much shorter than the limit set by the FEL bandwidth. The characteristics of the radiation averaged over all the shots are summarized in Table II.

In the second experimental session, corresponding to the images labeled beam B in Figs. 3-5, the electron beam (Table I, column B) was extracted closer to maximum compression, the two beamlets were completely overlapping, and the fringe contrast was higher than case A. Space charge effects cause the indentation of the phase space, as shown in Fig. 3( $\left.\mathrm{a}^{\prime}\right)$. In this case, almost all spectrometer shots are clearly double peaked. The separation of the peaks in the case shown in Fig. 4, second row, was $\Delta \lambda=$ $23.85 \mathrm{~nm}$. The FROG data are characterized by a neat
TABLE II. Radiation characteristics: $\Delta \lambda_{\mathrm{FR}}$ is the separation in wavelength measured by the FROG, $\Delta t_{\mathrm{rms}}$ the total pulse width, $V$ the visibility of the fringe, $V=\left(E_{\max }-E_{\min }\right) /\left(E_{\max }+E_{\min }\right)$, and $\Delta T$ the time separation between the fringes. All quantities are averaged over all the shots.

\begin{tabular}{lcccc}
\hline \hline Beam & $\Delta \lambda_{\mathrm{FR}}(\mathrm{nm})$ & $\Delta t_{\mathrm{rms}}(\mathrm{fs})$ & $V(\%)$ & $\Delta T(\mathrm{fs})$ \\
\hline A & $14 \pm 5$ & $196 \pm 25$ & 35 & $137 \pm 18$ \\
B & $16.6 \pm 4.1$ & $76.9 \pm 5.4$ & 85 & $110 \pm 26$ \\
\hline \hline
\end{tabular}

two-color spectrum with $\Delta \lambda=18.62 \mathrm{~nm}$, corresponding to an energy separation value of $\Delta E=1.1 \mathrm{MeV}$. The average spectral $\mathrm{rms}$ width of the single lines was $\Delta \lambda_{1,2}=$ $3.2 \pm 0.7 \mathrm{~nm}$. A Fourier limited pulse with this bandwidth should have a characteristics time scale of about $500 \mathrm{fs}$. The measured time structure of the whole pulse is instead noticeably shorter, with the fringe separation $\Delta T=91 \mathrm{fs}$, the average value over all the events being $\Delta T=110 \pm$ $26 \mathrm{fs}$. The fringe rms width is $\Delta t_{f, \text { rms }}=22.5 \mathrm{fs}$ with average value $\Delta t_{f, \text { rms }}=31.5 \mathrm{fs}$. When only two different pulses are used in this scheme, the expected temporal modulation is sinusoidal so that the rms width of the power temporal spikes is expected to be about one-third of their relative spacing. The experimental averaged values of the fringe separation $\Delta T$ (fs) of both working points agree with the previsions in Fig. 1, confirming the analytical scaling.

In this Letter we presented experimental analysis of the SASE emission of two simultaneous electron bunches separated in energy by a relative difference larger than $\rho$. The output radiation pulses, as measured by FROG diagnostics, present a time structure not limited by the single beam amplifier bandwidth. The method, which has been applied to the SPARC FEL for the generation of IR radiation with a regular short time modulation, can be extrapolated to the production of subfemtosecond pulses in the x-ray's range. A larger number $N$ of electron bunches could in principle be used to further shorten the duration of the pulses, taking advantage of the gratinglike effect. The development of such schemes is of significant importance since it offers a promising avenue for the femtosecond to attosecond science and nanotechnology at short wavelength FEL.

We wish to acknowledge the entire SPARC team for the long hours spent in the control room, making this work possible.

[1] P. B. Corkum and F. Krausz, Nat. Phys. 3, 381 (2007).

[2] F. Krausz and M. Ivanov, Rev. Mod. Phys. 81, 163 (2009).

[3] C. M. Guenther, B. Pfau, R. Mitzner, B. Siemer et al., Nat. Photonics 5, 99 (2011).

[4] J. Mauritsson, P. Johnsson, E. Mansten, M. Swoboda, T. Ruchon, A. L'Huillier, and K. J. Schafer, Phys. Rev. Lett. 100, 073003 (2008). 
[5] M. F. Kling and M. J. J. Vrakking, Annu. Rev. Phys. Chem. 59, 463 (2008).

[6] L. J.P. Ament, M. van Veenendaal, T.P. Devereaux, J. P. Hill, and J. van den Brink, Rev. Mod. Phys. 83, 705 (2011).

[7] A. McPherson, G. Gibson, H. Jara, U. Johann, T. S. Luk, I. A. McIntyre, K. Boyer, and C. K. Rhodes, J. Opt. Soc. Am. B 4, 595 (1987).

[8] K. P. Singh et al., Phys. Rev. Lett. 104, 023001 (2010).

[9] W. Ackermann et al., Nat. Photonics 1, 336 (2007).

[10] P. Emma et al., Nat. Photonics 4, 641 (2010).

[11] H. Tanaka et al., Nat. Photonics 6, 540 (2012).

[12] E. Allaria et al., Nat. Photonics 6, 699 (2012).

[13] R. Bonifacio, C. Pellegrini, and L. Narducci, Opt. Commun. 50, 373 (1984).

[14] R. Bonifacio, L. De Salvo, P. Pierini, N. Piovella, and C. Pellegrini, Phys. Rev. Lett. 73, 70 (1994); E. L. Saldin, E. A. Schneidmiller, and M. V. Yurkov, Opt. Commun. 281, 1179 (2008).

[15] J. Ronsenzweig et al., Nucl. Instrum. Methods Phys. Res., Sect. A 593, 39 (2008).

[16] Y. Ding et al., Phys. Rev. Lett. 102, 254801 (2009).

[17] E. L. Saldin, E. A. Schneidmiller, and M. V. Yurkov, Phys. Rev. ST Accel. Beams 9, 050702 (2006).

[18] L. Giannessi et al., Phys. Rev. Lett. 106, 144801 (2011).

[19] G. Marcus et al., Appl. Phys. Lett. 101, 134102 (2012).

[20] P. Emma, M. Cornacchia, K. Bane, Z. Huang, H. Schlarb, G. Stupakov, and D. Walz, Phys. Rev. Lett. 92, 074801 (2004).

[21] Y. Ding et al., Phys. Rev. Lett. 109, 254802 (2012).

[22] A. A. Lutman, R. Coffee, Y. Ding, Z. Huang, J. Krzywinski, T. Maxwell, M. Messerschmidt, and H.-D. Nuhn, Phys. Rev. Lett. 110, 134801 (2013).

[23] T. Watanabe, X. J. Wang, J. B. Murphy, J. Rose, Y. Shen, T. Tsang, L. Giannessi, P. Musumeci, and S. Reiche, Phys. Rev. Lett. 98, 034802 (2007).

[24] L. Giannessi et al., Phys. Rev. Lett. 110, 044801 (2013).

[25] G. DeNinno, B. Mahieu, E. Allaria, L. Giannessi, and S. Spampinati, Phys. Rev. Lett. 110, 064801 (2013).
[26] E. Allaria, F. Bencivenga, R. Borghes, and F. Capotondi, "Two-Color Pump-Probe Experiments with Twin-PulseSeed XUV Free Electron Lasers" (to be published).

[27] B. Mahieu, E. Allaria, D. Castronovo, and M. B. Danailov, arXiv:1306.1470.

[28] A. A. Zholents and W. M. Fawley, Phys. Rev. Lett. 92, 224801 (2004); E. L. Saldin, E. A. Schneidmiller, and M. V. Yurkov, Opt. Commun. 239, 161 (2004).

[29] G. Stupakov, Phys. Rev. Lett. 102, 074801 (2009).

[30] A. A. Zholents and G. Penn, Nucl. Instrum. Methods Phys. Res., Sect. A 612, 254 (2010).

[31] D. Xiang, Z. Huang, and G. Stupakov, Phys. Rev. ST Accel. Beams 12, 060701 (2009).

[32] N. R. Thompson and B. W. J. McNeil, Phys. Rev. Lett. 100, 203901 (2008).

[33] D. J. Dunning, B. W. J. McNeil, and N. R. Thompson, Phys. Rev. Lett. 110, 104801 (2013).

[34] M. Ferrario, D. Alesini, M. P. Anania, A. Bacci et al., Nucl. Instrum. Methods Phys. Res., Sect. A 309, 183 (2013).

[35] L. Giannessi, D. Alesini, P. Antici et al., Phys. Rev. ST Accel. Beams 14, 060712 (2011).

[36] M. Boscolo, M. Ferrario, I. Boscolo, F. Castelli, and S. Cialdi, Nucl. Instrum. Methods Phys. Res., Sect. A 577, 409 (2007).

[37] M. Ferrario et al., Phys. Rev. Lett. 104, 054801 (2010).

[38] M. Ferrario et al., Nucl. Instrum. Methods Phys. Res., Sect. A 637, S43 (2011).

[39] E. Chiadroni et al., Rev. Sci. Instrum. 84, 022703 (2013).

[40] M. Quattromini, M. Artioli, E. DiPalma, A. Petralia, and L. Giannessi, Phys. Rev. ST Accel. Beams 15, 080704 (2012).

[41] T-STEP is an upgraded version of PARMELA, see L. M. Young, Los Alamos National Laboratory Report No. LA-UR-96-1835.

[42] S. Reiche, Nucl. Instrum. Methods Phys. Res., Sect. A 429, 243 (1999). 